\title{
IMPLEMENTATION OF AUTODESK REVIT® IN DESIGN OF WATER TREATMENT PLANTS
}

\author{
HOSSAM KAMAL $^{1} \&$ MOHAMED BAHGAT ${ }^{2}$ \\ ${ }^{1}$ Advridis Ltd., UK \\ ${ }^{2}$ International Environmental Technology INTECH-HA, Egypt
}

\begin{abstract}
Design of water treatment plant is one of the challenging engineering design sectors. This engineering multi-disciplinary field implies the interaction of different scientific efforts, besides the management and economic aspects. The traditional 2D design workflow compromises many risks of time and effort waste and miss-estimated quantities, time, and cost. Although, BIM techniques present an efficient 6D solution for many construction projects, their applications in such sophisticated electromechanical-based projects deserve a lot of efforts to be adapted. The present work is the result of collaborative work between the BIM teams in the two companies to adapt Autodesk AEC ${ }^{\circledR}$ package to the design of both wastewater and potable water treatment plant. The two teams carried out five successive projects to establish a standard workflow for the implementation of BIM techniques, based on Autodesk Revit ${ }^{\circledR}$, in direct filtration, extended aeration, and sequential batch reactor Egyptian treatment plants. The results of the implementation of BIM techniques in water treatment plant design show the reduction of design time up to $67 \%$, the reduction of man power up to $75 \%$, increased accuracy of BOQ lists up to $94 \%$, and transfer of project planning procedure to semiautomated schedules.
\end{abstract}

Keywords: water treatment plant design, BIM techniques, Autodesk Revit ${ }$.

\section{INTRODUCTION}

The design and construction of water treatment plants represent an interesting challenge of the engineering design entities. In April 2020, the International Water Association (IWA) defines the major trends impacting the water industry in the next decade as [1]:

1. Acting on the mounting effects of extreme weather events;

2. Protecting agricultural production;

3. Reusing wastewater to support a circular economy;

4. A customer-led revolution;

5. Smart and intelligent network technologies.

All these trends implies the utilization of enhanced optimised design concepts and effective smart tools.

BIM helps project stakeholders to visualize all the project components in a simulated environment. The obtained model is a digital representation of the building that helps project stakeholders to extract needed information and take decisions during the project planning, design, construction, and operation processes [2]. The implementation of BIM in utility design requires the creation of new workflow that facilitates the work and exploits the new software tools. Dodge Data and Analytics illustrated the importance and impacts of implementation of BIM in the "Business Value of BIM for Water project" report [3]. In 2017, the project of "Advance Works for Shek Wu Hui Sewage Treatment Works - Further Expansion Phase 1A and Sewerage Works at Ping Che Road" was awarded the Autodesk Hong Kong BIM Awards [4].

The present work is the fruit of cooperation between Adviridis Ltd. (UK) and International Environmental Technology INTECH-HA (Egypt) to found a BIM unit and to 
build the team and capabilities in 3D modelling and design process. INTECH-HA is one of the pioneer EPC companies in Egypt and MENA region in water treatment. They have elaborated more than 200 projects using the traditional 2D CAD design. On the other hand, Adviridis Ltd. is specialized in smart solutions for environmental design. This task was carried out in the framework of complete business process reengineering of ITECH-HA. This reengineering process includes BIM unit, procurement database creation, and workflow management recreation. In this paper, we shed lights on the BIM system transformation. After recruiting the starting BIM team of three engineers, the BIM unit creation project starts with a pilot project of a wastewater treatment plant of capacity $20,000 \mathrm{~m}^{3} /$ day designed on the advanced sequential batch reactor (ASBR) technology. Then, the team is extended to six BIM engineers and two other projects were carried out. Finally, the BIM unit of eight engineers carried out three simultaneous projects with three different design technologies.

The main outcomes of this cooperation are:

1. the reduction of design time up to $67 \%$;

2. the reduction of man power up to $75 \%$; and

3. increased accuracy of BOQ lists up to $94 \%$.

From our point of view, the present work represent novelty in three folds:

1. The adoption of BIM procedures in one of the developing countries "Egypt" in different infrastructure projects.

2. The implementation of BIM workflow as a part business process reengineering procedure

3. The adaptation of Autodesk Revit ${ }^{\circledR}$ software in electromechanical-based projects.

The present paper starts by discussing the starting challenges. Then, the implemented workflow is introduced. Finally, the outcomes and the impacts on the business process are depicted. Generally, the present work shows the outcomes of the first phase of business process reengineering in engineering design. The future phases will widen the implementation of BIM in the project management procedures in the same company.

\section{CHALLENGES OF CREATION OF BIM THINKING}

To start a new business depending on a new technology is a hard job. However, it is an easy job compared to introducing the same new technology to a business entity that have already been working for more than two decades using traditional technologies. At the planning phase of this transformation project, the challenges were classified in three axes:

1. Personal workflow management

2. Technology adaptation

\subsection{Challenges of workflow management}

The first step in dealing with the axis of human resources is to evaluate present resources and their management protocol. The actual case was that the company consists of four sectors; engineering, projects, installations, and procurement. The main concern was the engineering sector. This sector was consisting of a general director, two directors of mechanical and electrical design, five electrical designers, eleven mechanical designers, and seven draftsmen. The structure design was considered as a part of project sector. The instrumentation and control design was considered as a part of installation sector. The workflow management starts from a process design producing process flow diagram (PFD). 
Then, the mechanical designer proposes the general arrangement and preliminary piping and instrumentation diagram (P\&ID). Then, both the mechanical and the electrical designer start working on detail design. After that, the civil guides are produced and are sent to the structure designer. In all these processes, the responsibility of the designer is to sketch her/his design and send it to a draftsman. The draftsman draws the 2D plans and sections and print them to be reviewed by the designer.

Within that environment of work, some challenges were sported:

- the designer spent about $55 \%$ of job time in reviewing the printed drawings.

- the senior designers had lost their ability to work on CAD software or to follow-up the capabilities of new releases.

- the draftsmen took about 18 months of successive work to understand the specific types of machines and terminology (decanter, scrapper, fine screen, etc.).

- the structure design was lagged after the mechanical design and should verified from the mechanical engineer manually.

- the electrical engineer should recalculate (or redesign) the after revision of mechanical and structure.

- the designs loop was iterated at least three times to arrive to the final design submitted for approval.

- Both engineers and draftsmen were adapting the 2D thinking in building the plans, projections, and sections. While 3D prospective was built only if a presentation was required for special purpose.

- The BOQs were counted manually.

- The cost and time of construction were calculated on spreadsheets depending on manual data entry.

- The psychological impact of introducing new technology with new workflow was huge on both engineers and draftsmen specially with the fear of losing their jobs or authorities during hard economic environment.

\subsection{Challenges of technology adaptation}

The water treatment processes depend on wide variety of equipment. Some of these equipment are on-the-shelf ones like pumps, motors, generators, ... etc. However, the core equipment of the treatment processes are not common and they are made unit-by-unit according to the plant design parameters. This implies that the item model library is continuously revised and updated in each project. The preliminary evaluation considered that about $40-25 \%$ of equipment models should be rebuilt in each project. Besides, the item that has a certain function has different designs according to supplier. This implies that in each new project the same item may be built at least three times and the dimensions and arrangement are modified accordingly. Fig. 1 shows the clarifier bridge from two suppliers for the same plant. One of the major challenges of water treatment plant design arrangement is the limited available land relative to the required capacity. For example, the WWTPs of Port Fouad and Dar Elsalam have the same capacity of $20,000 \mathrm{~m}^{3} /$ day and the same technology of extended aeration, but the available area of Port Fouad WWTP is $98.788 \mathrm{~m}^{2}$ while that of Dar Elsalam is $145.512 \mathrm{~m}^{2}$. This implies changing of $45 \%$ of the used equipment to achieve the required output. 

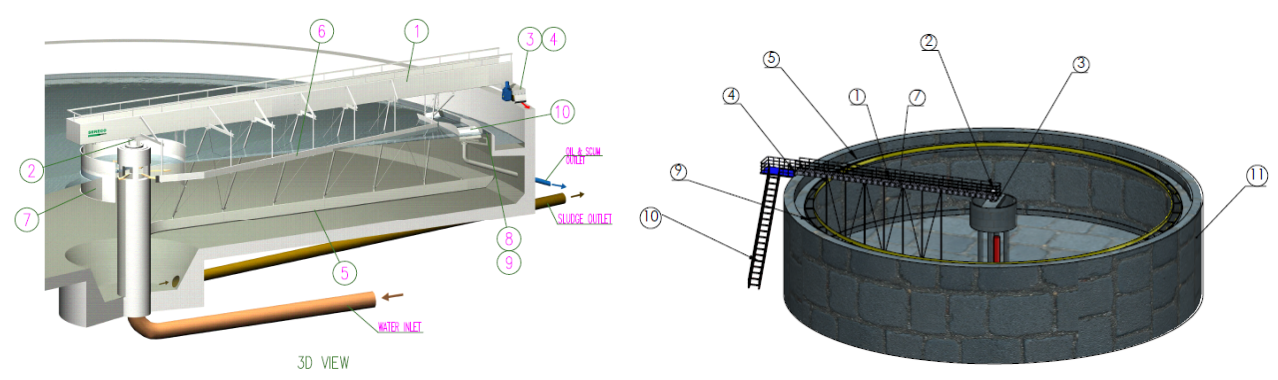

Figure 1: Different structure of scrapper of primary sedimentation tank.

\section{THE RE-ENGINEERED BIM WORKFLOW}

The transformation project starts by the analysis of current situation and building the issue register to quantify the business process waste types and sources. Root-cause analysis with Pareto chart are built and they lead the team to achieve a project of business process reengineering of the engineering sector. This project establishes the new system of building information modeling (BIM) in the International Environmental Technologies Company (INTECH). This system is realized by constructing a new unit; named Technical Support Unit (TSU). TSU includes two sections:

1. Information management section (IMS); and

2. Modeling and drafting section (MDS).

The project starts by specifying requirements and concluded by achieving a pilot project under the new system. The business process concept of work is to create a dynamic teamwork that can support both engineering and tendering processes.

The project objectives are set as:

1. Coordinate the RFI, RFQ, and RFP (in collaboration with Tender Department):

- Simplify the RFI process with a standardized workflow that maintains transparency for the entire team.

- Efficiently create, coordinate, and manage all submittals in a single document library. Initiate requests, manage reviews and approvals, and sync critical information for easy access.

2. Project document management

- Access to drawings and documents: Ensure all project teams have access to the information that they need on whatever device they are working on. Powerful search capabilities, a single viewer for plans, models and photos helps to keep the whole team on the same page.

- Precise construction document control: Standardize approval workflows, control access to information, and maintain version history.

- Centralized document management: Bring drawings, models, specifications, RFIs, photos, and markups together in a single document management platform.

3. Coordinate the work attribution to human resources (in collaboration with Engineering Department)

4. Perform quality assurance and quality control of engineering design processes and activities. 
5. Review of constructability and detect clashing and errors of design in real time.

6. Manage the urgent changes of design occurred during construction process.

7. Construction data and analytics

8. Perform the closing procedure of projects including documentation, archiving, and experience transfer.

9. Organize the paper-form document archiving system.

10. Construct and maintain an efficient electronic archiving system.

11. Drafting and drawing of project plans.

12. Building of component libraries to standardize and facilitate work.

13. Training/continuous training of workforce on new systems.

Fig. 2 depicts the structure of the created TSU and the assigned objectives to each position of the unit.

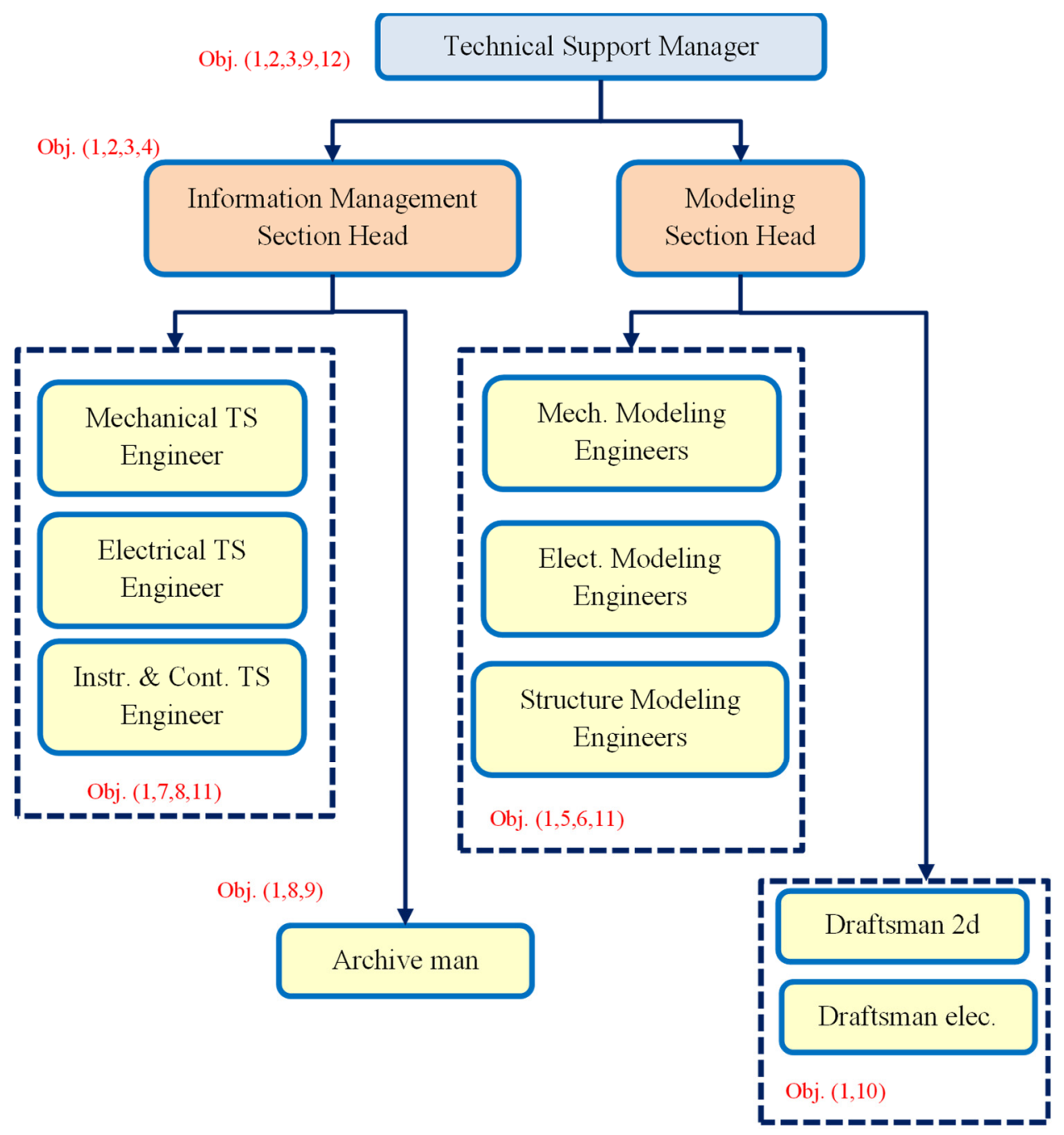

Figure 2: The structure of the created technical support unit. 
The pilot project was the Tuna Elgabal wastewater treatment plant (WWTP) having a capacity of $20,000 \mathrm{~m}^{3} /$ day and employing the technology of extended aeration of Bioworks $\mathrm{GmbH}$. The plant was designed using the traditional methods of 2D modelling. The design activities lasted 89 working days (including design revisions and consultant approvals) by two mechanical engineers, one electrical engineer, one control engineer, one instrumentation engineer. The plant included 17 types of main equipment (fine screens, coarse screens, mixers, sludge mixers, aeration tanks, electrical generators, etc.) and 14 types of supporting equipment (valves, sensors, cables, administration building, etc.). After recruiting three BIM engineers, the pilot project started on parallel tracks:

1. Training of two mechanical engineers and one electrical engineer on the BIM concepts and Autodesk Revit $\AA$ software.

2. Training of three draftsmen on the Autodesk Revit ${ }^{\circledR}$ software.

3. Training of newly recruited BIM engineers on the water treatment concepts.

4. Collection of equipment catalogues and drawings.

5. Upgrade of available PCs and procurement of new PCs.

6. Purchase of software packages and infrastructure components.

The pilot project lasted 67 working days as the design had been already verified. The three draftsmen succeeded to deal with BIM concepts and to build the item models on the Autodesk Revit ${ }^{\circledR}$ family builder. This success was essential from the psychological point of view as it encouraged all the draftsmen and engineers to support and join the new business process.

After completing the pilot project, the transformation team was built and the training of the rest of junior and senior engineers and draftsmen was carried out during 6 weeks on three groups to avoid the interruption of the original workflow.

In order to evaluate the transformation process, four other new projects were selected to be designed using BIM concepts and workflow. One of these projects was carried out in parallel tracks; traditional and BIM. While the other three projects were elaborated completely in BIM unit. Fig. 3 illustrates some examples of the plants modelled by Autodesk Revit ${ }^{\circledR}$. These projects had different design technologies:

1. Tahta WWTP of capacity $20,000 \mathrm{~m}^{3} /$ day and employing the technology of extended aeration of Bioworks $\mathrm{GmbH}$. This project lasted 43 working days including design verification and consultant approvals.

2. Assuite potable water treatment plant of capacity of $40,000 \mathrm{~m}^{3} /$ day employing the technology of lamella filtration and monolithic filter floors. This project lasted 51 working days including design verification and consultant approvals.

3. October potable water treatment plant (phase 3/4) of capacity of $400,000 \mathrm{~m}^{3} /$ day employing the technology of lamella filtration and monolithic filter floors. This project lasted 68 working days. This project was considered as a milestone for INTECH-HA company as their experience in potable water treatment plants was limited to the medium size plants of $40,000 \mathrm{~m}^{3} /$ day. Their previous plant of medium size took 109 working days because of the overwhelming details of lamella and filter nozzles.

4. New Capital WWTP of capacity $250,000 \mathrm{~m}^{3} /$ day and employing the technology of advanced sequential batch reactor (ASBR). The design activities of this project lasted 46 working days despite its huge challenges. This project was a challenge because of the large capacity of the plant and because that many technologies were employed like two different types of odour control systems, two types of chlorination systems, GRP covers for main process tanks, and many others. 

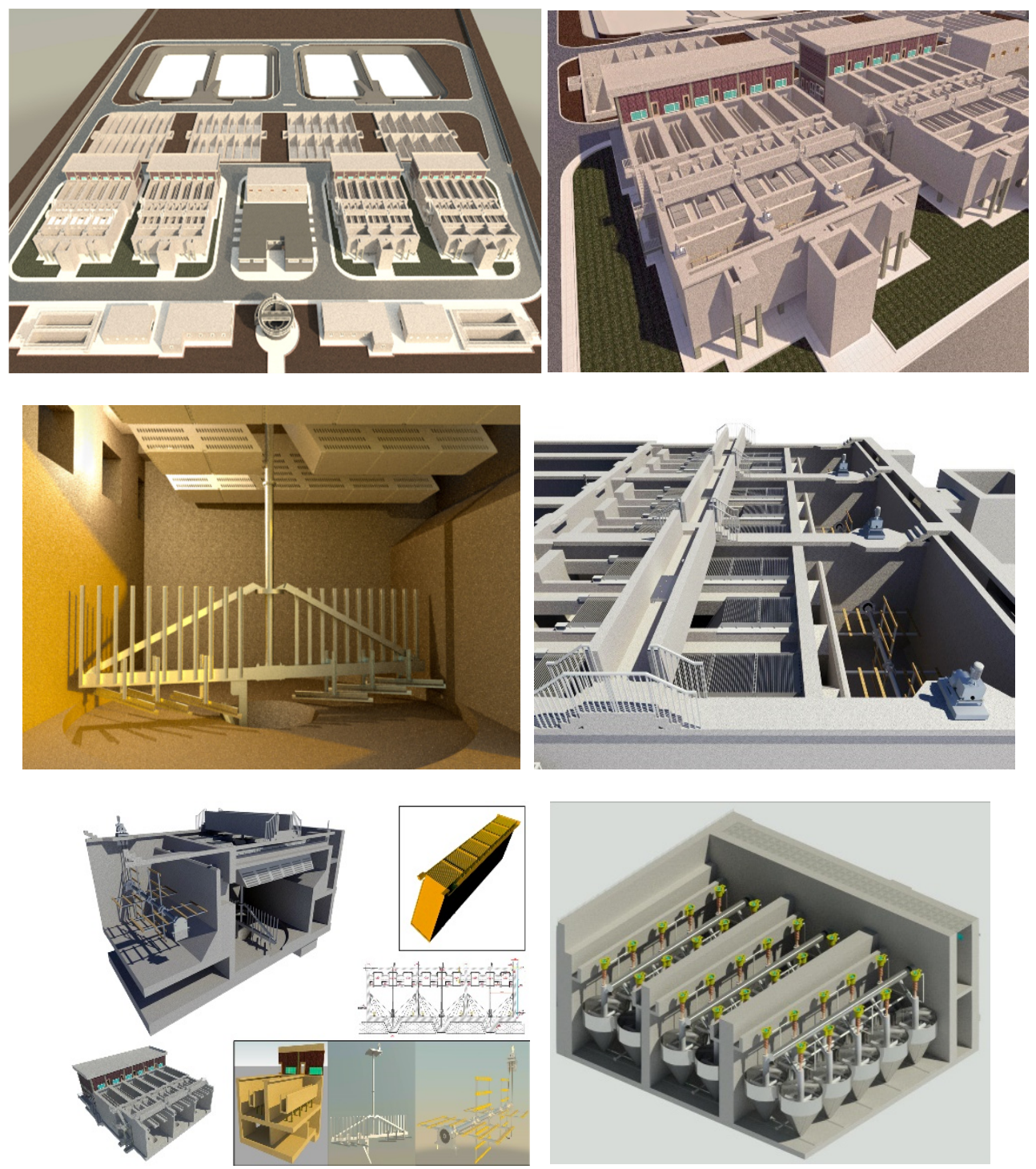

Figure 3: Some examples of the plants modelled by Autodesk Revit ${ }^{\circledR}$.

\section{THE BIM TRANSFORMATION EXPERIENCE}

After completing the business process reengineering process of BIM transformation, we can conclude our experience in the following points:

1. The results of the implementation of BIM techniques in water treatment plant design show the reduction of design time up to $67 \%$, the reduction of man power up to $65 \%$.

2. One of the major contributions of BIM system is automated and accurate elaboration of bill of quantities (BOQ). The accuracy of BOQ lists is increased up to 94\%. In traditional $2 \mathrm{D}$ design there is no way but counting items manually. The BIM-based workflow eliminates the job of counting engineer. 
3. BIM is known as $6 \mathrm{D}$ system as it incorporates cost, time, and as built information. In our present case we succeeded to fully benefit of cost information as the information extracted from Autodesk Revit ${ }^{\circledR}$ software is merged with that of the supplier database and the cost analysis is elaborated.

4. On the other hand, the utilization of time and installation information will be considered in the future phase as this job needs the inclusion of the other sectors of the company.

5. The Autodesk Revit ${ }^{\mathbb{R}}$ software is excellent in architecture-based design. However, in the electromechanical-based designs it could not be used as stand-alone. The workflow should include the Autodesk Civil $3 \mathrm{D}^{\circledR}$ software specially for piping isometric.

6. The psychological aspects of the transformation process are essential for the success of the process. The resistance of the section heads and directors was remarkable. This was eased after the achievement of the pilot project and realization of the fact that BIM concepts will promote their work. For the draftsmen, the BIM training was successful for five out of seven and we managed to keep the two failed draftsmen in the team.

7. As the Egyptian market is not fully accustomed to BIM concepts, we didn't fully eliminate the traditional workflow. We keep the two draftsmen who cannot comply with BIM concepts to elaborate the electrical single line diagrams, the mechanical P\&ID, and the digitization of paper drawings.

\section{ACKNOWLEDGEMENTS}

By the end of this work, we'd like to present our deep thanks and gratitude to the teams of Adviridis Ltd. (UK) and International Environmental Technology INTECH-HA (Egypt) for their time and efforts.

\section{REFERENCES}

[1] Wennberg, C., 5 Major Trends Impacting the Water Industry in the Next Decade, IWA, 22 Apr. 2020. iwa-network.org/five-major-challenges-and-emerging-trendsimpacting-the-water-industry-in-the-next-decade/.

[2] Azhar, S., Khalfan, M. \& Maqsood, T., Building information modelling (BIM): Now and beyond. Construction Economics and Building, 12(4), pp. 15-28, 2012.

[3] Jones, S.A. (ed.), SmartMarket Report: Business Value of BIM for Water Project, Dodge Data \& Analytics, 2018.

[4] Söbke, H., Theiler, M., Tauscher, E. \& Smarsly, K., BIM-based Description of Wastewater Treatment Plants, 2018.

[5] Riffat, R., Fundamentals of Wastewater Treatment and Engineering, CRC Press, 2013. 